\title{
Transmission eigenchannels from nonequilibrium Green's functions
}

\author{
Paulsson, Magnus; Brandbyge, Mads
}

Published in:

Physical Review B Condensed Matter

Link to article, DOI:

10.1103/PhysRevB.76.115117

Publication date:

2007

Document Version

Publisher's PDF, also known as Version of record

Link back to DTU Orbit

Citation (APA):

Paulsson, M., \& Brandbyge, M. (2007). Transmission eigenchannels from nonequilibrium Green's functions. Physical Review B Condensed Matter, 76(11), 115117. https://doi.org/10.1103/PhysRevB.76.115117

\section{General rights}

Copyright and moral rights for the publications made accessible in the public portal are retained by the authors and/or other copyright owners and it is a condition of accessing publications that users recognise and abide by the legal requirements associated with these rights.

- Users may download and print one copy of any publication from the public portal for the purpose of private study or research.

- You may not further distribute the material or use it for any profit-making activity or commercial gain

- You may freely distribute the URL identifying the publication in the public portal

If you believe that this document breaches copyright please contact us providing details, and we will remove access to the work immediately and investigate your claim. 


\title{
Transmission eigenchannels from nonequilibrium Green's functions
}

\author{
Magnus Paulsson ${ }^{1,2,3, *}$ and Mads Brandbyge ${ }^{1, \dagger}$ \\ ${ }^{1}$ Department of Micro- and Nanotechnology (MIC), Technical University of Denmark (DTU), \\ $\emptyset$ rsteds Plads, DK-2800 Lyngby, Denmark \\ ${ }^{2}$ Department of Electronics, Toyama University, Gofuku, Toyama 930-8555, Japan \\ ${ }^{3}$ Division of Physics, School of Pure and Applied Natural Sciences, University of Kalmar, 39182 Kalmar, Sweden
}

(Received 13 February 2007; revised manuscript received 11 June 2007; published 18 September 2007)

The concept of transmission eigenchannels is described in a tight-binding nonequilibrium Green's function (NEGF) framework. A simple procedure for calculating the eigenchannels is derived using only the properties of the device subspace and quantities normally available in a NEGF calculation. The method is exemplified by visualization in real space of the eigenchannels for three different molecular and atomic wires.

DOI: 10.1103/PhysRevB.76.115117

PACS number(s): 73.23.Ad, 73.63.Nm, 73.63.Rt

\section{INTRODUCTION}

Electronic transport properties of atomic-scale conductors have been investigated intensively in the past decade. ${ }^{1,2}$ Examples of interest include molecular wires connected to metal electrodes, atomic metal wires, and nanotubes. Firstprinciples transport calculations on these systems give results that are, in general, difficult to interpret due to the multichannel nature of the scattering problem and the fact that the scattering states are generated from the atomic valence orbitals. The free-electron type of reasoning normally used in mesoscopic quantum transport is, thus, not adequate. It is, therefore, useful to analyze the conduction in terms of eigenchannels. Eigenchannels are particular scattering states ${ }^{3}$ with a well-defined transmission probability, $0 \leqslant T_{n} \leqslant 1$, where the individual eigenchannel transmissions add up to the total transmission $T=\Sigma_{n} T_{n}$. In addition to being useful for analyzing theoretical calculations, the eigenchannel transmissions may be obtained experimentally (i) with superconducting electrodes connecting the atomic-scale conductor, as shown by Scheer et al. ${ }^{4}$ or (ii) from shot noise measurements, ${ }^{1}$ where information about the individual channel contributions can be obtained since the Fano factor involves the sum ${ }^{1,5}$ $\Sigma_{n} T_{n}\left(1-T_{n}\right) / \Sigma_{n} T_{n}$.

Eigenchannels have previously been calculated for atomic metal wires ${ }^{6,7}$ and molecular contacts ${ }^{8}$ by directly solving for the scattering states in the leads. This type of analysis breaks up the transmission into the "nonmixing" channels ${ }^{3,9,10}$ and gives an intuitive picture of electron transport. The ability to plot the eigenchannel wave functions is especially useful, since it gives a direct spatially resolved picture of the orbitals involved in the transport. Another possibility is to consider projections of eigenchannel wave functions onto, for example, molecular orbitals.

An increasingly popular theoretical approach to calculate transport properties is the nonequilibrium Green's function (NEGF) formalism. ${ }^{11}$ This is normally used in combination with a tight-binding type or linear combination of atomic orbitals electronic structure description. ${ }^{12-15}$ In this approach, it is straightforward to calculate the single particle (retarded) Green's function (matrix) including coupling to the infinite electrodes by introducing self-energies. The Green's function is, thus, the fundamental quantity in these calculations, and scattering states are normally not considered. Interpretation of the results in terms of scattering states is, therefore, nontrivial. ${ }^{16}$ In contrast, the scattering states are the fundamental quantity in approaches based on the LippmannSchwinger equation, where jellium models are normally used to describe the electrodes. ${ }^{17-20}$

The aim of this paper is to show how the eigenchannels can be easily generated within the NEGF approach without solving for the scattering states in the leads. The eigenchannel wave functions are here obtained directly from quantities readily available in the NEGF calculation, e.g., the retarded Green's function matrix, $G_{D}$ of the device region, and the $\Gamma_{L, R}$ matrices describing the coupling of the device region to the two electrodes ("left" $L$ and "right" $R$, see Fig. 1). In the case of atomistically defined electrodes, this approach is especially advantageous since solving for the scattering states requires calculating the Bloch waves in the electrodes (complex band structure), which may be a nontrivial numerical task for large unit cells. ${ }^{21}$ Related to our approach is the so-called (left and/or right) open "channel functions" of Inglesfield et al. based on the "embedding potential" in the real-space formulation ${ }^{22}$ and the Korringa-Kohn-Rostokerbased formulation by Bagrets et al. ${ }^{23,24}$ In addition to providing a simple way to calculate the eigenchannels, the method presented here offers an intuitive understanding of the oneparticle NEGF equations; e.g., it may be used to understand propensity rules for the effect of phonon scattering on the electronic transport. ${ }^{25,26}$

The paper is organized as follows. Section II starts with the definition of eigenchannels and a summary of the standard one-particle NEGF equations. Our method to obtain the eigenchannels without solving for the full transmission matrix is then derived. The usefulness of the eigenchannels is

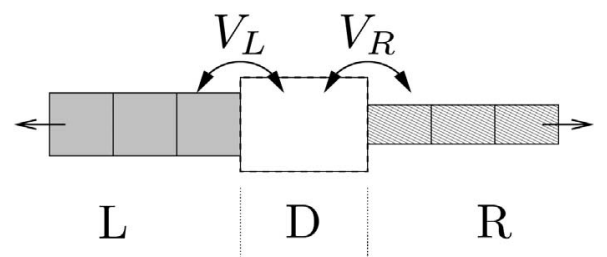

FIG. 1. The generic two probe system which couples the left $(L)$ and right $(R)$ leads through an intermediate "device" region $(D)$. 
illustrated in Sec. III, with three examples where the eigenchannels are calculated for molecular and atomic wires connected to gold electrodes using a first-principles density functional method.

\section{EIGENCHANNELS}

We consider transport through a device region $D$ coupled to two semi-infinite leads, left and right $(L, R)$, and limit ourselves to leads built from periodic cells. The solutions in the corresponding infinite leads are Bloch states $\left|u_{l}\right\rangle\left(\left|u_{r}\right\rangle\right)$ denoted by band index $l(r)$ for the left (right) lead. In order to obtain the transmission amplitude matrix $\mathbf{t}_{r, l}$ at a given energy $E$, we consider the solutions to the Schrödinger equation, $\left|\Psi_{l}\right\rangle$, with scattering boundary conditions and incoming waves in the left lead,

$$
\begin{gathered}
\left|\Psi_{l}\right\rangle=\frac{\left|u_{l}\right\rangle}{\sqrt{v_{l}}}+\text { reflected, left lead, } \\
\left|\Psi_{l}\right\rangle=\sum_{r} \frac{\left|u_{r}\right\rangle}{\sqrt{v_{r}}} \mathbf{t}_{r, l}+\text { decaying, right lead, }
\end{gathered}
$$

where $\left|u_{\rangle}\right\rangle$is an incoming Bloch wave from the left lead, i.e., right-moving, with energy $E$ and group velocity $v_{l}>0$, and $\left|u_{r}\right\rangle$ is an outgoing Bloch wave $\left(v_{r}>0\right)$. At a certain energy, the number of such incoming channels, $N_{L}(E)$, is determined by the band structure of the lead. Likewise, there are $N_{R}(E)$ such outgoing channels on the right. In Eq. (1), we explicitly state the flux normalization by dividing the Bloch state by $\sqrt{v_{l}}$, where the Bloch waves $\left|u_{l}\right\rangle$ are normalized in the conventional manner over the infinite leads ${ }^{31}\left\langle u_{l}(k) \mid u_{l^{\prime}}\left(k^{\prime}\right)\right\rangle$ $=\delta_{l l^{\prime}} \delta\left(k-k^{\prime}\right)$. The reflected and transmitted parts may contain evanescent decaying waves which have zero velocity, and for those states, we use normal integral normalization. With these considerations, we find that the flux-normalized states fulfill the normalization,

$$
\frac{1}{\sqrt{v_{l}}}\left\langle u_{l}(k) \mid u_{l^{\prime}}\left(k^{\prime}\right)\right\rangle \frac{1}{\sqrt{v_{l^{\prime}}}}=\hbar \delta_{l l^{\prime}} \delta\left(E-E^{\prime}\right)
$$

since the velocity is related to the energy as $v_{l}$ $=1 / \hbar d \varepsilon_{l}(k) / d k$. The scattering states generated from the flux-normalized incoming waves will also be flux normalized, see the Appendix,

$$
\left\langle\Psi_{l} \mid \Psi_{l^{\prime}}\right\rangle=\hbar \delta_{l l^{\prime}} \delta\left(E-E^{\prime}\right),
$$

and likewise for $r, r^{\prime}$ while $\left\langle\Psi_{l} \mid \Psi_{r}\right\rangle=0$.

The advantage of using flux-normalized states is that we can make any unitary transformation between the incoming scattering states from the left lead at a particular energy,

$$
\left|w_{l}\right\rangle=\sum_{l^{\prime}} \frac{\left|u_{l^{\prime}}\right\rangle}{\sqrt{v_{l}}}\left(\mathbf{U}_{L}\right)_{l^{\prime}, l}
$$

These new states will again solve the Schrödinger equation, only contain incoming waves in the left lead, and be flux normalized. However, the mix of Bloch waves will no longer have Bloch symmetry. Naturally, we can apply a similar transformation of the outgoing right channels with $\mathbf{U}_{R}$. Especially, we can choose the transformations $\left(\mathbf{U}_{L}, \mathbf{U}_{R}\right)$ such that the transmission matrix $\mathbf{t}$ becomes a diagonal matrix, ${ }^{9} \mathbf{t}_{e}$, at a specific energy

$$
\mathbf{t}_{e}=\mathbf{U}_{R}^{\dagger} \mathbf{t} \mathbf{U}_{L}=\operatorname{diag}\left(\sqrt{T_{1}}, \sqrt{T_{2}}, \ldots\right) .
$$

This corresponds to a singular value decomposition of the transmission amplitude matrix, or a diagonalization of the Hermitian (left to right) transmission probability matrix,

$$
\mathbf{T}_{e}=\mathbf{t}_{e}^{\dagger} \mathbf{t}_{e}=\mathbf{U}_{L}^{\dagger} \mathbf{t}^{\dagger} \mathbf{t} \mathbf{U}_{L}=\operatorname{diag}\left(T_{1}, T_{2}, \ldots\right),
$$

where there will be a maximum of $\min \left(N_{L}(E), N_{R}(E)\right)$ nonzero eigenvalues $0 \leqslant T_{\alpha} \leqslant 1$.

The diagonalization of the transmission matrix defines the transmission eigenchannels $\left|\Phi_{\alpha}\right\rangle$ of the system as the unitary mix of left incoming flux-normalized scattering states ("channels") given by $\mathbf{U}_{L}$; i.e., the left eigenchannels are given by $\left|\Phi_{\alpha}\right\rangle=\Sigma_{l^{\prime}}\left|\Psi_{l^{\prime}}\right\rangle\left(\mathbf{U}_{L}\right)_{l^{\prime}, \alpha^{\prime}}$. The eigenchannels have the special property of being nonmixing in the sense that the transmission of a sum of 2 is the sum of individual fluxes or transmissions (equal for flux-normalized states). For example, consider the alternative channels given by the scattering states defined by the two first eigenchannels as $\left|u_{a}\right\rangle$ $=a_{1}\left|\Phi_{1}\right\rangle+a_{2}\left|\Phi_{2}\right\rangle$, with transmission $T_{a}=\left|a_{1}\right|^{2} T_{1}+\left|a_{2}\right|^{2} T_{2}$, and similarly, $\left|u_{b}\right\rangle$ with transmission $T_{b}$. Now the total transmission of $\left|u_{c}\right\rangle=\left|u_{a}\right\rangle+\left|u_{b}\right\rangle$ will be $T_{c}=T_{a}+T_{b}+I$, with the interference term $I=2 T_{1} \operatorname{Re}\left(a_{1} b_{1}^{*}\right)+2 T_{2} \operatorname{Re}\left(a_{2} b_{2}^{*}\right)$. It will, thus, not simply be the sum of the two transmissions, $T_{c} \neq T_{a}+T_{b}$. Since the purpose of this paper is to calculate the eigenchannels without solving for the complex band structure in the leads, we will use the fact that the eigenchannels also maximize the transmission through the device; i.e., the first eigenchannel from the left contact maximizes the transmission probability over the space of incoming states from the left, the next channel maximizes the transmission while being orthogonal to the first channel, etc.

In the following, we will, instead of flux normalization, make use of energy normalization $\left\langle\Psi_{n}(E) \mid \Psi_{m}\left(E^{\prime}\right)\right\rangle$ $=\delta_{n m} \delta\left(E-E^{\prime}\right)$, with the trivial difference from flux normalization being a factor $\sqrt{\hbar}$. Energy normalization is advantageous when working with energy resolved quantities since the natural continuous quantum number for this normalization is the energy. In addition, we can interpret the energynormalized states as a density of states; i.e., $\left|\Psi_{l}(x)\right|^{2}$ $=\left|\left\langle x \mid \Psi_{l}\right\rangle\right|^{2}$ is the projected (local) density of states at $x$.

\section{A. Preliminaries}

The one-particle Hamiltonian for the tight-binding scattering problem shown in Fig. 1 can be written

$$
H=H_{0}+V=H_{L}+H_{D}+H_{R}+V_{L}+V_{R},
$$

where the isolated leads $H_{L, R}$ and device $H_{D}$ are coupled 
together by the interactions between leads and device $(V$ $=V_{L}+V_{R}$ ) without any direct coupling between the leads. Using projection operators onto the device $P_{D}$ and left and/or right leads $P_{L, R}\left(I=P_{L}+P_{D}+P_{R}\right)$, we can also define $\tau_{L, R}$ $=P_{D} V_{L, R} P_{L, R}$, where $V_{L, R}=\tau_{L, R}+\tau_{L, R}^{\dagger}$. The rest of this section provides a short summary of the standard definitions of oneparticle Green's functions, self-energies, and the notation we will use throughout the paper. ${ }^{11,27}$

From the definition of the retarded Green's function (operator) for the whole system, we can find the expansion of the Green's function in an eigenbasis, $H\left|\Psi_{m}(E)\right\rangle=E\left|\Psi_{m}(E)\right\rangle$,

$$
G(E)=(E+i \delta-H)^{-1}=\int d E^{\prime} \sum_{m} \frac{\left|\Psi_{m}\left(E^{\prime}\right)\right\rangle\left\langle\Psi_{m}\left(E^{\prime}\right)\right|}{E+i \delta-E^{\prime}},
$$

where the infinitesimal imaginary part $\delta=0^{+}$ensures that the Green's function yields the retarded response of the system. The device part of the Green's function can further be written

$$
G_{D}=\left(E+i \delta-H_{D}-\Sigma_{L}-\Sigma_{R}\right)^{-1},
$$

where we have introduced the self-energies, $\Sigma_{L, R}$ $=\tau_{L, R} g_{L, R} \tau_{L, R}^{\dagger}$, given by the Green's functions of the isolated leads $g_{L, R}=\left(E+i \delta-H_{L, R}\right)^{-1}$. In addition to the Green's function, the spectral functions $A(E)=i\left(G-G^{\dagger}\right)$ and $a_{L, R}=i\left(g_{L, R}\right.$ $\left.-g_{L, R}^{\dagger}\right)$ and broadening $\Gamma_{L, R}=i\left(\Sigma_{L, R}-\Sigma_{L, R}^{\dagger}\right)=\tau_{L, R} a_{L, R} \tau_{L, R}^{\dagger}$ will be needed. The fact that these matrices live on different subspaces will be used repeatedly, e.g., $\Gamma_{L}=P_{D} \Gamma_{L} P_{D}$, etc.

For the scattering problem, see Fig. 1, we know that the time independent discrete Schrödinger equation has a complete set of solutions. These solutions can be divided into a continuous set of solutions $\left|\Psi_{n}(E)\right\rangle$ (where there may be several solutions at any given energy) and localized states $\left|\Psi_{m}^{\mathrm{Loc}}\right\rangle$ with energy $E_{m}^{\mathrm{Loc}}$. We use the energy as the continuous quantum number together with a discrete quantum number $n$, i.e., subbands. Since we are only interested in the transport properties, we will, from here on, ignore localized states. ${ }^{32}$

In the following, we will describe a method to determine the eigenchannel scattering states inside the device region using the information contained in $G_{D}, \Gamma_{L}$, and $\Gamma_{R}$. The spectral function $A$ is a central quantity in the following discussion. It can be obtained from the expansion of the retarded Green's function in eigenfunctions to the Hamiltonian [Eq. (8)],

$$
A(E)=i\left[G(E)-G^{\dagger}(E)\right]=2 \pi \sum_{n}\left|\Psi_{n}(E)\right\rangle\left\langle\Psi_{n}(E)\right| .
$$

\section{B. Scattering states from the leads}

We may choose to express the solutions to the Schrödinger equation as solutions consisting of waves originating in the left or right lead. These scattering states can be generated from the spectral function, as will be shown here. Decomposing the spectral function of the device $A_{D}$ $=P_{D} A P_{D}$, using Eq. (9), we find

$$
\begin{aligned}
A_{D} & =i\left(G_{D}-G_{D}^{\dagger}\right)=i G_{D}\left(G_{D}^{\dagger-1}-G_{D}^{-1}\right) G_{D}^{\dagger} \\
& =G_{D} \Gamma_{L} G_{D}^{\dagger}+G_{D} \Gamma_{R} G_{D}^{\dagger},
\end{aligned}
$$

where we, in the following, wish to show that $A_{L, R}$ $=G_{D} \Gamma_{L, R} G_{D}^{\dagger}$ is generated by the scattering states with incoming waves in the left (right) lead.

Viewing the coupling between device and leads as a perturbation, we can start with a set of orthogonal and normalized eigenfunctions, $\left|\tilde{u}_{l}\right\rangle$, of the isolated left lead (and similarly for the right), which are totally reflected solutions since they are solutions for the isolated semi-infinite leads. From these states, the full solutions $\left|\Psi_{l}\right\rangle$ can be generated,

$$
\left|\Psi_{l}\right\rangle=G V_{L}\left|\widetilde{u}_{l}\right\rangle+\left|\tilde{u}_{l}\right\rangle
$$

The response given by the retarded Green's function only contains waves traveling outward from the device region. These solutions to the Schrödinger equation, thus, have the required property of being incoming from the left lead. In addition, the solutions are energy normalized and orthogonal; see the Appendix.

We can then express the device part of the spectral function from the solutions generated by Eq. (12),

$$
\begin{aligned}
A_{L} & =2 \pi \sum_{l} P_{D}\left|\Psi_{l}\right\rangle\left\langle\Psi_{l}\right| P_{D} \\
& =2 \pi \sum_{l} P_{D}\left(G V_{L}\left|\widetilde{u}_{l}\right\rangle+\left|\widetilde{u}_{l}\right\rangle\right)\left(\left\langle\widetilde{u}_{l}\right|+\left\langle\widetilde{u}_{l}\right| V_{L}^{\dagger} G^{\dagger}\right) P_{D} \\
& =P_{D} G V_{L} a_{L} V_{L}^{\dagger} G^{\dagger} P_{D}=G_{D} \Gamma_{L} G_{D}^{\dagger},
\end{aligned}
$$

where we have used Eq. (10) for the whole system and for the isolated lead. Apart from rederiving Eq. (11), we have proven that the two parts of the device spectral function $A_{L, R}=G_{D} \Gamma_{L, R} G_{D}^{\dagger}$ are built up of scattering states originating from the respective leads. This immediately leads to the well known formula for the density matrix in nonequilibrium (excluding localized states)

$$
\rho=\frac{1}{2 \pi} \int_{-\infty}^{\infty}\left[f_{L}(E) A_{L}(E)+f_{R}(E) A_{R}(E)\right] \mathrm{d} E
$$

where $f_{L, R}$ is the Fermi function of the leads.

\section{Current operator}

To find the eigenchannels of the system, we will need the current operator. The number of electrons in lead $R$ is described by the projection operator $P_{R}$. The operator for current into $R$ is, thus, determined as the time derivative of $P_{R}$,

$$
\hat{J}_{R}=2 e \dot{P}_{R}=\frac{i 2 e}{\hbar}\left[H, P_{R}\right]=\frac{i 2 e}{\hbar}\left(\tau_{R}-\tau_{R}^{\dagger}\right)
$$

where we have evaluated the commutator using the Hamiltonian, Eq. (7), and included a factor of 2 for spin.

The current into lead $R$ due to the scattering state with energy $E,\left|\Psi_{l}\right\rangle$, originating from $L$ from the original incoming (standing) wave $\left|\widetilde{u}_{l}\right\rangle$, can be written 


$$
j_{l l}=\left\langle\Psi_{l}|\hat{J}| \Psi_{l}\right\rangle .
$$

To simplify this expression, we extract the right lead part of the wave function using the Lippmann-Schwinger Eq. (A2), which gives

$$
P_{R}\left|\Psi_{l}\right\rangle=P_{R} G_{0} V\left|\Psi_{l}\right\rangle+P_{R}\left|\widetilde{u}_{l}\right\rangle=g_{R} \tau_{R}^{\dagger}\left|\Psi_{l}\right\rangle,
$$

where all quantities are evaluated at energy $E$. The current carried by the scattering state $\left|\Psi_{l}\right\rangle$ is then given by

$$
\begin{aligned}
j_{l l} & =\frac{i 2 e}{\hbar}\left\langle\Psi_{l}\left|\tau_{R}-\tau_{R}^{\dagger}\right| \Psi_{l}\right\rangle \\
& =\frac{i 2 e}{\hbar}\left(\left\langle\Psi_{l}\left|\tau_{R} g_{R} \tau_{R}^{\dagger}\right| \Psi_{l}\right\rangle-\left\langle\Psi_{l}\left|\tau_{R} g_{R}^{\dagger} \tau_{R}^{\dagger}\right| \Psi_{l}\right\rangle\right) \\
& =\frac{e}{\pi \hbar} 2 \pi\left\langle\Psi_{l}\left|\Gamma_{R}\right| \Psi_{l}\right\rangle .
\end{aligned}
$$

Summing the current over all the orthogonal, energynormalized scattering states originating in $L$ at the specific energy yields the net current or total transmission and, thus, the Landauer formula,

$$
\begin{aligned}
j & =\sum_{l} J_{l l}=\frac{e}{\pi \hbar} 2 \pi \sum_{l}\left\langle\Psi_{l}\left|\Gamma_{R}\right| \Psi_{l}\right\rangle \\
& =\frac{e}{\pi \hbar} \operatorname{Tr}\left(A_{L} \Gamma_{R}\right)=\frac{e}{\pi \hbar} \operatorname{Tr}\left(G_{D} \Gamma_{L} G_{D}^{\dagger} \Gamma_{R}\right) .
\end{aligned}
$$

From Eq. (18), we notice that the transmission probability for any scattering state from the left $\left|\Psi_{l}\right\rangle$ is given by $2 \pi\left\langle\Psi_{l}\left|\Gamma_{R}\right| \Psi_{l}\right\rangle$. The transmission probability matrix can, therefore, be written

$$
T_{l^{\prime} l}=2 \pi\left\langle\Psi_{l^{\prime}}\left|\Gamma_{R}\right| \Psi_{l}\right\rangle,
$$

since we consider energy-normalized (flux normalized except for the factor of $\hbar$ ) scattering states, $|\Psi\rangle$, for which the current is equivalent to the transmission.

\section{Scattering states in the device region}

To find the eigenchannels from the left lead, $\left|\Phi_{l}\right\rangle$ $=\Sigma_{l^{\prime}}\left(\mathbf{U}_{L}\right)_{l l^{\prime}}\left|\Psi_{l^{\prime}}\right\rangle$, we need to diagonalize the transmission probability matrix. Since we do not, in this paper, explicitly calculate the Bloch states in the leads, we will diagonalize the transmission probability matrix in an abstract basis formed by incoming waves from the left lead. Another, equivalent, formulation is to find the eigenchannels by maximizing the transmission at a given energy while keeping the eigenchannels orthogonal $\left(\mathbf{U}_{L} \mathbf{U}_{L}^{\dagger}=\mathbf{1}\right)$,

$$
\max 2 \pi\left\langle\Phi_{l}\left|\Gamma_{R}\right| \Phi_{l}\right\rangle .
$$

The main problem in finding the eigenchannels is that we do not have access to the wave functions, Green's functions, or spectral functions of the entire system in a typical calculation. We, therefore, have to find a method using only properties from the device part of the system.

We will now show that optimizing the current by varying the scattering state over space spanned by the incoming

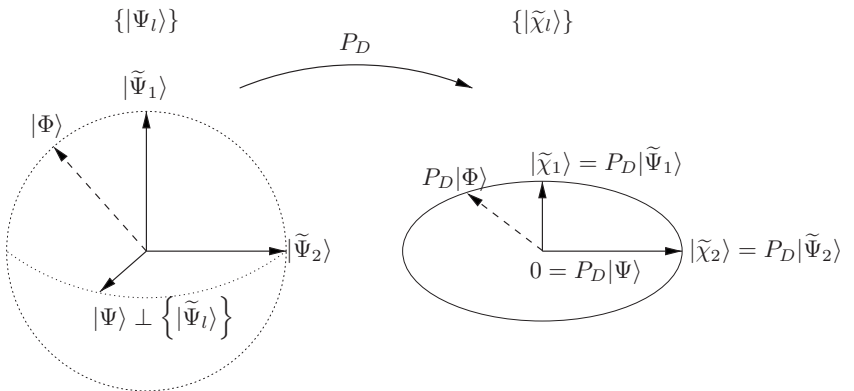

FIG. 2. Overview of the different states used in this paper. Note that the scattering states which are nonzero in the device subspace are all spanned by the $\left|\widetilde{\Psi}_{l}\right\rangle$ states. We can, therefore, find the eigenchannels by studying the wave functions spanned by the $\left|\tilde{\chi}_{l}\right\rangle$ $=P_{D}\left|\widetilde{\Psi}_{l}\right\rangle$.

states from the left lead, $\left\{\left|\Psi_{l}\right\rangle\right\}$, is equivalent to varying over the states $\left|\widetilde{\chi}_{l}\right\rangle$ defined on the device subspace; see Fig. 2. We start by diagonalizing the device part of the spectral function from the left lead,

$$
A_{L}(E)=\sum_{l}\left|\chi_{l}\right\rangle \lambda_{l}\left\langle\chi_{l}\left|=2 \pi \sum_{l}\right| \tilde{\chi}_{l}\right\rangle\left\langle\tilde{\chi}_{l}\right|,
$$

where the eigenvectors on the finite device space are orthonormal $\left\langle\chi_{l} \mid \chi_{l^{\prime}}\right\rangle=\delta_{l, l^{\prime}}$. Each $\left|\tilde{\chi}_{l}\right\rangle=\sqrt{\lambda_{l} / 2 \pi}\left|\chi_{l}\right\rangle$ with nonzero eigenvalues $\left(\lambda_{l} \neq 0\right)$ is the device part of a specific state $\left|\widetilde{\Psi}_{l}\right\rangle$, i.e., $\left|\widetilde{\chi}_{l}\right\rangle=P_{D}\left|\widetilde{\Psi}_{l}\right\rangle$, where the $\left|\widetilde{\Psi}_{l}\right\rangle$ states are normalized and orthogonal linear combinations of the scattering states $\left|\Psi_{l}\right\rangle$,

$$
\begin{aligned}
\left|\tilde{\chi}_{l}\right\rangle & =\sqrt{\frac{\lambda_{l}}{2 \pi}}\left|\chi_{l}\right\rangle=\frac{1}{\sqrt{2 \pi \lambda_{l}}} A_{L}(E)\left|\chi_{l}\right\rangle \\
& =P_{D} \sqrt{\frac{2 \pi}{\lambda_{l}}} \sum_{l^{\prime}}\left|\Psi_{l^{\prime}}\right\rangle\left\langle\Psi_{l^{\prime}} \mid \chi_{l}\right\rangle \equiv P_{D}\left|\tilde{\Psi}_{l}\right\rangle
\end{aligned}
$$

[using Eq. (13)], where we have defined

$$
\left|\widetilde{\Psi}_{l}\right\rangle=\sum_{l^{\prime}} \sqrt{\frac{2 \pi}{\lambda_{l^{\prime}}}}\left\langle\Psi_{l^{\prime}} \mid \chi_{l}\right\rangle\left|\Psi_{l^{\prime}}\right\rangle \equiv \sum_{l^{\prime}} W_{l l^{\prime}}\left|\Psi_{l^{\prime}}\right\rangle
$$

This shows that the states $\left|\widetilde{\Psi}_{l}\right\rangle$ are spanned by the incoming scattering states from the left lead. We further wish to show that these states are normalized in the same manner as the original scattering states; i.e., we want to show that $W_{m n}$ is unitary,

$$
\begin{aligned}
\sum_{n} W_{m n} W_{m^{\prime} n}^{*} & =\frac{2 \pi}{\sqrt{\lambda_{m} \lambda_{m^{\prime}}}} \sum_{n}\left\langle\chi_{m^{\prime}} \mid \Psi_{n}\right\rangle\left\langle\Psi_{n} \mid \chi_{m}\right\rangle \\
& =\frac{1}{\sqrt{\lambda_{m} \lambda_{m^{\prime}}}}\left\langle\chi_{m^{\prime}}\left|A_{L}\right| \chi_{m}\right\rangle \\
& =\frac{\lambda_{m}}{\sqrt{\lambda_{m} \lambda_{m^{\prime}}}}\left\langle\chi_{m^{\prime}} \mid \chi_{m}\right\rangle=\delta_{m m^{\prime}},
\end{aligned}
$$

using Eqs. (13) and (22).

In addition, any scattering states outside the space spanned by $\left|\widetilde{\Psi}_{n}\right\rangle$ are orthogonal to the device subspace. This 
can be seen from the fact that we can write the spectral function on the device subspace as

$$
A_{L}=2 \pi \sum_{l} P_{D}\left|\widetilde{\Psi}_{l}\right\rangle\left\langle\widetilde{\Psi}_{l}\left|P_{D}=2 \pi \sum_{l} P_{D}\right| \Psi_{l}\right\rangle\left\langle\Psi_{l}\right| P_{D} .
$$

Comparing the two equations reveals that $P_{D}|\Psi\rangle\langle\Psi| P_{D}$ must be zero for any $|\Psi\rangle$ which is orthogonal to the space spanned by $\left|\widetilde{\Psi}_{l}\right\rangle$.

In this section, we have shown that the wave functions $\left|\tilde{\chi}_{l}\right\rangle$ span the device part of any scattering state generated from lead $L$; see Fig. 2. To find the left eigenchannels, we can, therefore, maximize the current through the device with respect to a linear combination of $\left|\widetilde{\chi}_{l}\right\rangle$ instead of maximizing with respect to the full scattering states $\left|\Psi_{l}\right\rangle$. This is equivalent to diagonalizing the transmission matrix [Eq. (20)] in the basis formed by $\left|\tilde{\chi}_{l}\right\rangle$.

\section{E. Finding the eigenchannels}

In general, the basis $\left\{\left|e_{i}\right\rangle\right\}$ used in the calculations is nonorthogonal with the overlap matrix defined by $[\mathbf{S}]_{i j}=\left\langle e_{i} \mid e_{j}\right\rangle$. Although the eigenchannels may be calculated directly in this nonorthogonal basis, we will make use of Lövdin orthogonalization to simplify the algebra. The orthogonalized matrices (denoted by a bar) are given by $\overline{\boldsymbol{\Gamma}}_{R}=\mathbf{S}^{-1 / 2} \boldsymbol{\Gamma}_{R} \mathbf{S}^{-1 / 2}$ and $\overline{\mathbf{A}}_{L}=\mathbf{S}^{1 / 2} \mathbf{A}_{L} \mathbf{S}^{1 / 2}$. The eigenchannels obtained in the Lövdin orthogonalized basis can, at the end of the calculation, simply be transformed back into the nonorthogonal basis for further visualization or projection.

From the previous section, we learned that it is enough to diagonalize the transmission probability matrix [Eq. (20)] $T_{l^{\prime} l}=2 \pi\left\langle\widetilde{\chi}_{l^{\prime}}\left|\bar{\Gamma}_{R}\right| \widetilde{\chi}_{l}\right\rangle$ in the abstract basis $\left\{\left|\widetilde{\chi}_{l}\right\rangle\right\}$. To transform $2 \pi \bar{\Gamma}_{R}$ into the basis $\left\{\left|\widetilde{\chi}_{l}\right\rangle\right\}$, we first need to calculate the eigenvectors of $\bar{A}_{L}=\bar{G} \bar{\Gamma}_{L} \bar{G}^{\dagger}$,

$$
\sum_{n}\left[\overline{\mathbf{A}}_{L}\right]_{m n}[\mathbf{U}]_{n l}=\lambda_{l}[\mathbf{U}]_{m l},
$$

where $\mathbf{U}$ is unitary. We then obtain the transformation matrix to the $\left\{\left|\tilde{\chi}_{l}\right\rangle\right\}$ basis,

$$
[\tilde{\mathbf{U}}]_{m l}=\sqrt{\frac{\lambda_{l}}{2 \pi}}[\mathbf{U}]_{m l},
$$

which gives the explicit expression for the matrix we want to diagonalize

$$
T_{l^{\prime} l}=2 \pi\left\langle\widetilde{\chi}_{l^{\prime}}\left|\bar{\Gamma}_{R}\right| \widetilde{\chi}_{l}\right\rangle=2 \pi\left[\widetilde{\mathbf{U}}^{\dagger} \overline{\boldsymbol{\Gamma}}_{R} \widetilde{\mathbf{U}}\right]_{l^{\prime} l} .
$$

The eigenproblem is, therefore,

$$
\sum_{n} 2 \pi\left[\widetilde{\mathbf{U}}^{\dagger} \overline{\boldsymbol{\Gamma}}_{R} \tilde{\mathbf{U}}\right]_{m n}[\mathbf{c}]_{n \alpha}=T_{\alpha}[\mathbf{c}]_{m \alpha},
$$

where the eigenchannel vectors $\mathbf{c}_{\alpha}$ are given in the basis described by the columns of $\widetilde{\mathbf{U}}$, and the eigenvalues $T_{\alpha}$ are the transmission probabilities of the individual eigenchannels, $\alpha$. Finally, transforming back to the original nonorthogonal basis (from $\widetilde{\mathbf{U}}$ basis to the Löwdin basis, and from
Löwdin basis to normal nonorthogonal basis), we find that the eigenchannels on the device subspace are given by

$$
P_{D}\left|\Phi_{\alpha}\right\rangle=\sum_{i, n}\left[\mathbf{S}^{-1 / 2} \widetilde{\mathbf{U}}\right]_{i n}[\mathbf{c}]_{n \alpha}\left|e_{i}\right\rangle .
$$

Equations (27)-(31) provide a recipe for calculating the eigenchannels of a specific scattering problem using only properties available in standard NEGF calculations. In contrast to Ref. 16, these eigenchannels are well-defined scattering states calculated without approximations on the full device subspace.

It is interesting to note that the eigenchannels [Eq. (31)] are eigenvectors to $G \Gamma_{L} G^{\dagger} \Gamma_{R}$ (can be shown using the formalism presented above). This provides a simple method to obtain an idea about the eigenchannels. However, it is important to realize that the eigenchannel wave functions calculated from Eq. (31) are energy normalized; i.e., the amplitudes are well defined and can be compared between different eigenchannels. In contrast, the eigenvectors to $G \Gamma_{L} G^{\dagger} \Gamma_{R}$ may have any normalization and it is, therefore, not possible to compare amplitudes between different channels. Moreover, the energy-normalized scattering states Eq. (31) yield amplitudes which correspond to local density of states and are useful to plot, as will be shown in the next section.

\section{EIGENCHANNELS FOR ATOMIC AND MOLECULAR WIRES}

To exemplify the method developed in the previous section, we will use three different examples of atomic and molecular wires connected to gold electrodes. Using the TRANSIESTA (Ref. 12) extension of the SIESTA (Ref. 28) density functional theory (DFT) code, we have previously studied elastic and inelastic transport properties for the systems under consideration here: (i) atomic gold wires ${ }^{29}$ and the conjugated organic molecules, (ii) oligo-phenylene vinylene $\mathrm{e}^{30}$ (OPV), and (iii) oligo-phenylene ethynylene (OPE). ${ }^{30}$ The TRANSIESTA calculations on these systems were performed using DFT in the generalized gradient approximation using the Perdew-Burke-Ernzerhof functional. The semi-infinite leads connecting the device region was modeled using selfenergies in the NEGF method. A more detailed description of the calculational method may be found in Ref. 29.

Gold atomic wires have been realized and studied experimentally by several different techniques. The low bias elastic and inelastic (phonon scattering) transport are well characterized and understood; see Ref. 29, and references therein. The first eigenchannel (from left) at the Fermi energy is shown in Fig. 3 for a seven-atom gold chain. Not surprisingly, the majority of the transmission is carried by the first eigenchannel $\left(T_{1}=0.994\right)$, with only a small transmission for the other eigenchannels $\left(<10^{-5}\right)$. In addition, it is clear that the current through the wire is carried by the $6 s$ electrons forming a half-filled one-dimensional band, where the sign of the wave function changes by a factor of $i$ (right moving) along the wire. The only difference with the corresponding eigenchannel from the right (not shown) is the phase factor, which corresponds to a left-moving wave. 


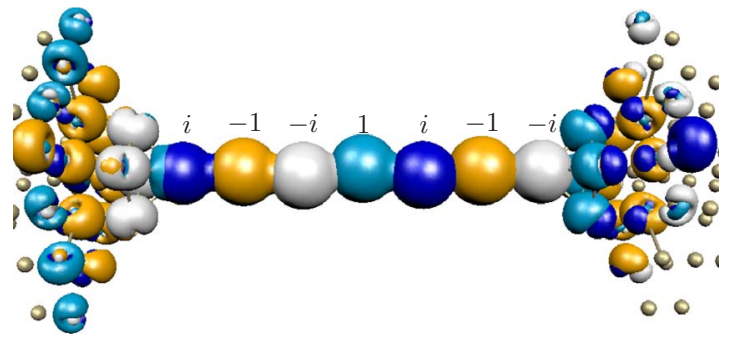

FIG. 3. (Color online) Left eigenchannel for a seven-atom Au atomic chain connected by four atom pyramids to $\mathrm{Au}$ (100) surfaces. The complex phase of the eigenchannel, indicated and shown in color, is similar that of a Bloch wave at the Fermi level in the infinite atomic gold chain (half-filled $6 s$ band).

For molecular wires, the experimental and theoretical understanding of electron transport are less well understood. The calculated transmission through the OPV molecule shown in Fig. 4 is 0.037 , and $100 \%$ of the transmission is carried through the first eigenchannel. Since the wave is almost totally reflected, the imaginary part of the wave function is too small to be seen in the figure. In the calculation, the thiol bonds to the hollow site on the Au (111) surface, and clearly shows that the conjugation of the molecule continues through the sulfur atom and that there is significant coupling to the gold leads.

To investigate an asymmetric case, we carried out calculations on an OPE molecule bound by a thiol to the left lead and with a tunneling barrier (hydrogen termination) to the right hand lead; see Fig. 5. The calculational details are the same as for the OPE molecule in Ref. 30. Because of the tunneling barrier, the transmission, $T_{t o t}=0.0026$, is lower than for the OPV molecule, and the left and right eigenchannels are considerably different. This can easily be understood by the large reflection at the right junction.

In the three examples described here, we find that the symmetry of the eigenchannels can be intuitively understood from the band structure of the corresponding infinite wires. For the gold wire, the $5 d$ band is below the Fermi energy, which is situated approximately at half filling of the $6 s$ band. The corresponding infinite wires (polymers) for the molecular wires have energy gaps at the Fermi energy. The eigenchannels, therefore, show the exponentially decaying solutions of the $\pi$-electron state in the complex band structure at the Fermi energy. ${ }^{21}$

\section{SUMMARY}

We have, in this paper, developed a method to calculate the scattering states corresponding to elastic eigenchannels.

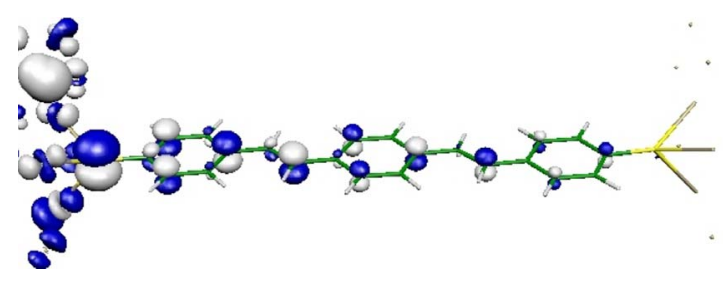

FIG. 4. (Color online) Left eigenchannel for an OPV molecule bound by thiols to the hollow sites on Au (111) surfaces. The colors correspond to the two different signs of the almost real-valued wave function.

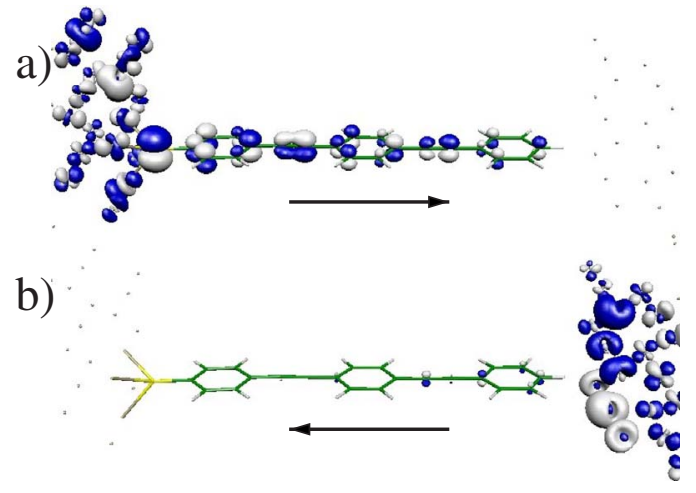

FIG. 5. (Color online) (a) Left and (b) right eigenchannels for an OPE molecule strongly bound by a thiol to the left surface and weakly interacting with the right lead.

The method is summarized in Eqs. (27)-(31), where the eigenchannels are found from quantities normally available in transport calculations using the NEGF technique. In addition, we show three brief examples of elastic scattering states calculated for molecular and atomic wires connected to three-dimensional contacts. The eigenchannels for these systems can be understood from the band structure of the infinite wires, providing an intuitive understanding.

The eigenchannels are useful in interpreting elastic electron transport through junctions. We believe that they will be especially useful in investigating the effect of the contacts between device and leads, e.g., binding site of the thiol bond on Au surfaces. In addition, the method gives a useful basis to understand the effects of phonon scattering on the conductance and their propensity rules..$^{25,26}$

\section{ACKNOWLEDGMENTS}

The authors would like to thank S. Datta, T. Frederiksen, C. Krag, and C. Rostgaard for useful discussions. This work, as part of the European Science Foundation EUROCORES Programme SASMEC, was supported by funds from the SNF and the EC 6th Framework Programme. Computational resources were provided by the Danish Center for Scientific Computations (DCSC).

\section{APPENDIX: ORTHOGONALITY OF SCATTERING STATES}

Viewing the Bloch states in the infinite, periodic leads, $\left|u_{l}\right\rangle$, as a starting point for perturbation theory, we can obtain the totally reflected solutions $\left|\widetilde{u}_{l}\right\rangle$ of a semi-infinite lead. In this case, the perturbation is the removal of the coupling between the periodic cells at the surface. Furthermore, the totally reflected states may again be used as the starting point in a perturbation calculation to obtain the full scattering states $\left|\Psi_{l, r}\right\rangle$. In this case, the perturbation is the device region and its coupling to the leads. Here, we will show that the perturbation expansion gives solutions that are orthogonal and normalized. To do this, we focus on the perturbation expansion of $\left|\Psi_{l}\right\rangle$ from $\left|\widetilde{u}_{l}\right\rangle$ and note that the same derivation may be used to obtain the $\left|\widetilde{u}_{l}\right\rangle$ from $\left|u_{l}\right\rangle$. 
Starting with a set of orthogonal and energy-normalized eigenfunctions $\left|\widetilde{u}_{n}(E)\right\rangle$ of the isolated leads $(n \in l, r)$, we generate the full scattering states $\left|\Psi_{n}(E)\right\rangle$,

$$
\left|\Psi_{n}(E)\right\rangle=G(E) V\left|\widetilde{u}_{n}(E)\right\rangle+\left|\widetilde{u}_{n}(E)\right\rangle,
$$

where $V=V_{L}+V_{R}$. The response given by the retarded Green's function only contains waves traveling outward from the device region. To show that the solutions generated in this way are normalized, we use the Lippmann-Schwinger equation

$$
\left|\Psi_{n}(E)\right\rangle=G_{0}(E) V\left|\Psi_{n}(E)\right\rangle+\left|\widetilde{u}_{n}(E)\right\rangle
$$

where the unperturbed Green's function is $G_{0}(E)=\left(E-H_{L}\right.$ $\left.-H_{R}-H_{D}+i \delta\right)^{-1}$. Together with Eq. (A1), we obtain

$$
\begin{aligned}
\left\langle\Psi_{n}(E) \mid \Psi_{n^{\prime}}\left(E^{\prime}\right)\right\rangle & \\
= & \left\langle\widetilde{u}_{n}(E) \mid \Psi_{n^{\prime}}\left(E^{\prime}\right)\right\rangle+\left\langle\widetilde{u}_{n}(E)\left|V^{\dagger} G^{\dagger}(E)\right| \Psi_{n^{\prime}}\left(E^{\prime}\right)\right\rangle \\
= & \left\langle\widetilde{u}_{n}(E) \mid \widetilde{u}_{n^{\prime}}\left(E^{\prime}\right)\right\rangle+\left\langle\widetilde{u}_{n}(E)\left|G_{0}\left(E^{\prime}\right) V\right| \Psi_{n^{\prime}}\left(E^{\prime}\right)\right\rangle \\
& +\left\langle\widetilde{u}_{n}(E)\left|V^{\dagger} G^{\dagger}(E)\right| \Psi_{n^{\prime}}\left(E^{\prime}\right)\right\rangle \\
= & \delta_{n, n^{\prime}} \delta\left(E-E^{\prime}\right)+\left\langle\widetilde{u}_{n}(E)|V| \Psi_{n^{\prime}}\left(E^{\prime}\right)\right\rangle \\
& \times\left(\frac{1}{E^{\prime}-E+i \delta}+\frac{1}{E-E^{\prime}-i \delta}\right) \\
= & \delta_{n, n^{\prime}} \delta\left(E-E^{\prime}\right),
\end{aligned}
$$

which show that the final scattering states $\left|\Psi_{n}\right\rangle$ are orthogonal and normalized.
*Present address: Division of Physics, School of Pure and Applied Natural Sciences, University of Kalmar, 39182 Kalmar, Sweden. magnus.paulsson@hik.se

†mbr@mic.dtu.dk

${ }^{1}$ N. Agraiit, A. L. Yeyati, and J. M. van Ruitenbeek, Phys. Rep. 377, 81 (2003).

${ }^{2}$ G. Cuniberti, G. Fagas, and K. Richter, Introducing Molecular Electronics (Springer, New York, 2005).

${ }^{3}$ M. Büttiker, IBM J. Res. Dev. 32, 63 (1988); eigenchannels are mentioned in Appendix C.

${ }^{4}$ E. Scheer, N. Agraït, J. C. Cuevas, A. L. Yeyati, B. Ludoph, A. Martin-Rodero, G. R. Bollinger, J. M. van Ruitenbeek, and C. Urbina, Nature (London) 394, 154 (1998).

${ }^{5}$ Y. M. Blanter and M. Büttiker, Phys. Rep. 336, 1 (2000).

${ }^{6}$ N. Kobayashi, M. Brandbyge, and M. Tsukada, Phys. Rev. B 62, 8430 (2000).

${ }^{7}$ M. Brandbyge and N. Lorente, Scanning Probe Microscopies Beyond Imaging (Wiley-VCH, New York, 2006), Chap. 15, pp. 77-97.

${ }^{8}$ J. Taylor, M. Brandbyge, and K. Stokbro, Phys. Rev. B 68, 121101(R) (2003).

${ }^{9}$ M. Brandbyge, M. R. Sørensen, and K. W. Jacobsen, Phys. Rev. B 56, 14956 (1997).

${ }^{10}$ J. C. Cuevas, A. L. Yeyati, and A. Martín-Rodero, Phys. Rev. Lett. 80, 1066 (1998).

${ }^{11}$ S. Datta, Electronic Transport in Mesoscopic Systems (Cambridge University Press, Cambridge, 1995).

${ }^{12}$ M. Brandbyge, J.-L. Mozos, P. Ordejón, J. Taylor, and K. Stokbro, Phys. Rev. B 65, 165401 (2002).

${ }^{13}$ P. Damle, A. W. Ghosh, and S. Datta, Chem. Phys. 281, 171 (2002).

${ }^{14}$ A. R. Rocha, V. M. Garcia-Suarez, S. Bailey, C. Lambert, J. Ferrer, and S. Sanvito, Phys. Rev. B 73, 085414 (2006), URL: http://link.aps.org/abstract/PRB/v73/e085414
${ }^{15}$ J. J. Palacios, A. J. Pérez-Jiménez, E. Louis, E. SanFabián, and J. A. Vergés, Phys. Rev. B 66, 035322 (2002).

${ }^{16}$ D. Jacob and J. J. Palacios, Phys. Rev. B 73, 075429 (2006).

${ }^{17}$ N. D. Lang, Phys. Rev. B 52, 5335 (1995).

${ }^{18}$ K. Hirose and M. Tsukada, Phys. Rev. B 51, 5278 (1995).

${ }^{19}$ M. Paulsson and S. Stafström, Phys. Rev. B 64, 035416 (2001).

${ }^{20}$ M. Di Ventra and N. D. Lang, Phys. Rev. B 65, 045402 (2002).

${ }^{21}$ J. K. Tomfohr and O. F. Sankey, Phys. Rev. B 65, 245105 (2002).

${ }^{22}$ J. E. Inglesfield, S. Crampin, and H. Ishida, Phys. Rev. B 71, 155120 (2005).

${ }^{23}$ A. Bagrets, N. Papanikolaou, and I. Mertig, Phys. Rev. B 75, 235448 (2007).

${ }^{24}$ A. Bagrets, N. Papanikolaou, and I. Mertig, Phys. Rev. B 73, 045428 (2006).

${ }^{25}$ M. Paulsson, T. Frederiksen, and M. Brandbyge, Phys. Rev. B 72, 201101(R) (2005).

${ }^{26}$ M. Paulsson, T. Frederiksen, and M. Brandbyge (unpublished).

${ }^{27}$ M. Paulsson, arXiv:cond-mat/0210519 (unpublished).

${ }^{28}$ P. Ordejon, E. Artacho, and J. M. Soler, Phys. Rev. B 53, R10441 (1996).

${ }^{29}$ T. Frederiksen, M. Paulsson, M. Brandbyge, and A.-P. Jauho, Phys. Rev. B 75, 205413 (2007).

${ }^{30}$ M. Paulsson, T. Frederiksen, and M. Brandbyge, Nano Lett. 6, 258 (2006).

${ }^{31}$ The flux $v_{l}$ is the band velocity divided by the unit-cell length, $L$, while the left lead $k$ vector is in units of the inverse unit-cell lengths; likewise for $R$. Alternatively, $k$ has a unit of inverse length while the $v$ 's are in units of velocity. In any case, the unit of the flux-normalized wave functions is square root of time.

${ }^{32}$ Since we are interested in scattering states around the Fermi energy, we may choose an energy arbitrarily close to the Fermi energy, where there are no localized states. 